\title{
Analysis and Prediction of Atmospheric Pollution of Shijiazhuang City in Hebei based on the Grey Theory
}

\author{
D.M. HUANG ${ }^{1} \&$ T. XIAO ${ }^{1}$ \\ ${ }^{1}$ College of Sciences, Agricultural University of Hebei Province, Hebei
}

KEYWORD: atmospheric pollution; gray correlation degree analysis; simulation; prediction, GM $(1,1)$

ABSTRACT: Atmospheric pollution is influenced by many uncertain factors, such as the content of pollutants, wind, etc., Therefore, one given environment system can be considered as a grey information system. In this paper, According to the monitoring data of atmospheric pollution in Shijiazhuang city of Hebei from 2006 to 2011,the data of air pollution of Shijiazhuang in Hebei is treated by used nondimensionalization approaches, and then the impact factors of atmospheric pollution of Shijiazhuang city in Hebei are firstly sorted by using the gray correlation degree analysis. Next the grey $G M(1,1)$ model of the atmospheric pollution of Shijiazhuang city in Hebei is formulated. By using the achieved models both to simulate and to predict the atmospheric pollution and the trend of the atmospheric pollution in the next two years of Shijiazhuang city in Hebei is predicted based on $G M(1,1)$ model. Finally, according to the prediction data given by the discussed models, the reasonable suggestions for alleviating the atmospheric pollution are proposed.

\section{INTRODUCTION}

With the adverse effect caused by industrialization and urbanization, the environment crisis is received more and more attention today. In this sense, citizens living in given regions, particularly metropolis, are being choked by poisonous gases emit by industrial plants and running vehicles. Because these toxic gases does not dilute instantly, PM10 and PM2.5 are known as the primary pollutant and simultaneously air is being worsened. These inhalable particles can be easily found in major cities and featured with the high concentrations and long residence periods. The analysis and study of the particle concentrations have been received a large number of attention. Therefore, evaluation of air quality and its relevant environmental treatments are conducted ${ }^{[1-8]}$.

The grey system theory intends to tackle uncertain systems featured with the small sample and poor information. All random variables are considered as grey scale Within a certain range of time and space. Also, the random process is treated as the grey process with variation of time. By processing information and by extracting the valuable part, the evolution, mechanism and dynamics of the system can be described and surveillance. The grey system $G M(1,1)$ model have their advantages to deal with the real-world problems. Hence, the grey system theory is widely applied in different fields, such as agriculture, health science and biology. Moreover, such atmosphere factors as TSP, $\mathrm{pH}$ level and $\mathrm{NO}_{2}$ has been partially studied with mathematical knowledge. However, researching the correlation between the atmospheric environment and inhalable particles still remains in qualitative analysis.

This paper organizes as follows. First, the sequence of factors determining the environmental quality of Shijiazhuang city in Hebei is investigated by employing the grey correlation degree analysis; by nondimensionalizing the origin data of the atmosphere of Shijiazhuang city in Hebei, the $G M(1,1)$ model for the environmental quality are formulated to simulate the atmosphere pollution.

Then, By using the achieved models both to simulate and to predict the atmospheric pollution and the trend of the atmospheric pollution in the next two years of Shijiazhuang city in Hebei is predicted based on $\operatorname{GM}(1,1)$ model. Finally, according the obtained mathematical conclusions, the 
corresponding suggestions for the environmental protection improvement are given. Matlab is used to conduct the computation in this paper.

\section{CAUSES OF THE ATMOSPHERE POLLUTION AND DETERMINATION FOR MAIN IMPACT FACTORS}

\section{The impact factors of the atmosphere pollution}

The extent of the atmosphere pollution is determined a variety of factors, such as wind and pollutant emission, but the correlation of each factor of atmosphere pollution may vary. Then, according to the reports published by China National Environment Monitoring Center and China Statistical Yearbook, the inhalable particles $P M 10$, sulfur dioxide $\mathrm{SO}_{2}$ and nitrogen dioxide $\mathrm{NO}_{2}$ are identified as impact factors. Secondly, the total amount of investment (OvallIEI) and the total amount of air pollutant emissions (TotalIEE) from the air pollution control is two main influencing factors (such as table 1).

Table 1. The environment quality of Shijiazhuang in Hebei and the investment of the air quality treatment( OvallIEI).

Time Days $\quad \mathrm{PM}_{10} \mathrm{SO}_{2} \quad \mathrm{NO}_{2} \quad$ OvallIEI TotalIEE

\begin{tabular}{lllllll}
\hline 2006 & 78 & 0.142 & 0.044 & 0.039 & 137799 & ------- \\
2007 & 76 & 0.128 & 0.043 & 0,035 & 137800 & 48036 \\
2008 & 65 & 0.116 & 0.046 & 0.031 & 97174 & 37558 \\
2009 & 47 & 0.104 & 0.045 & 0.035 & 91106 & 50779 \\
2010 & 46 & 0.098 & 0.054 & 0.041 & 82054 & 56324 \\
2011 & 45 & 0.099 & 0.052 & 0.041 & 156828 & ------
\end{tabular}

* Days :days Below level two for the air quality;

* OvallIEI :overall investment for environment improvement (millions);

* TotalIEE :total of industrial exhaust emission (billion cubic meters) .

The nondimensionalization for the data of the impact factor of the atmosphere pollutant

The main impact factors are determined as 5 factors affecting the environmental quality in Shijiazhuang of Hebei, which is mentioned in Table.1. However, the mechanism of factors and correlation among them are still unknown. In order to guarantee the accuracy of the calculation, the nondimensionalization of the different factors is essential and the commonly used nondimensionalization approaches go as follows. Through comparative analysis, we choose

$x_{i}(k)=\frac{y_{i}(k)-m}{M-m}, \quad i=0,1,2, \mathrm{~L}, N$.

where $y_{i}(k)$ denotes Measured value of the five impact factors mentioned above, namely $P M 10$, $\mathrm{SO}_{2}, \mathrm{NO}_{2}$, the overall investment and the total emission; $\mathrm{M}$ and $m$ are their maximum and minimum respectively; $N=5$.

\section{The grey relational degree computation}

The grey relational degree calculation steps are as follows ${ }^{[1]}$.

a). Assume the nondimensionalized matrix obtained from the (original data matrix) is

$$
X=\left[\begin{array}{cccc}
x_{0}^{(0)}(1) & x_{1}^{(0)}(1) & \mathrm{L} & x_{n}^{(0)}(1) \\
x_{0}^{(0)}(2) & x_{1}^{(0)}(2) & \mathrm{L} & x_{n}^{(0)}(2) \\
\mathrm{M} & \mathrm{M} & \mathrm{M} & \mathrm{M} \\
x_{0}^{(0)}(N) & x_{1}^{(0)}(N) & \mathrm{L} & x_{n}^{(0)}(N)
\end{array}\right] ;
$$


b). Obtain the maximal difference and minimal difference in the sequence

We obtain the absolute difference between the first row and other rows and then arrive the absolute difference matrix is

$\Delta=\left[\begin{array}{cccc}\Delta_{01}(1) & \Delta_{02}(1) & \mathrm{L} & \Delta_{0 n}(1) \\ \Delta_{01}(2) & \Delta_{02}(2) & \mathrm{L} & \Delta_{0 n}(2) \\ \mathrm{M} & \mathrm{M} & \mathrm{M} & \mathrm{M} \\ \Delta_{01}(N) & \Delta_{02}(N) & \mathrm{L} & \Delta_{0 n}(N)\end{array}\right]$

Where $\Delta_{0 i}(k)=\left|x_{0}^{(0)}(k)-x_{i}^{(0)}(k)\right|$,

$i=0,1,2, \mathrm{~L}, n ; \quad k=0,1,2, \mathrm{~L}, N$.

The maximal difference and minimal difference are the maximal value and the minimal value in the absolute difference matrix :

$\Delta(\max )=\max _{i}\left\{\max _{k}\left\{\Delta_{0 i}(k)\right\}\right\} ;$
$\Delta(\min )=\min _{i}\left\{\min _{k}\left\{\Delta_{0 i}(k)\right\}\right\}$.

c). Calculate the correlation coefficient

After the original data nondimensionalized, the grey correlation degree between $x_{0}$ and $x_{i}$ at $\mathrm{k}$ is:

$\xi_{0 i}=\frac{\Delta(\min )+\rho \Delta(\max )}{\Delta_{0 i}(k)+\rho \Delta(\max )}$,

where $\rho$ is in $(0,1$,$) . it is usually assumed to be \rho=0.5$. The grey correlation degree is the average value of correlation coefficients,

$\gamma_{0 i}=\frac{1}{N} \sum_{k=1}^{N} \xi_{0 i}(k)$.

\section{The determination of the grey correlation degree of the factors affecting the air pollution}

According to the statistical data of the pollution and management of the atmosphere in Hebei, we take the day number of air quality below the second level as our analysis object, that is the standard sequence $y_{0}$; Also, we choose $P M 10, \mathrm{SO}_{2}, \mathrm{NO}_{2}$, the overall investment for environment improvement (OvallIEI) and the total amount of industrial exhaust emission (TotalIEE) as the impact factor $y_{i}, i=1,2, \mathrm{~L}, 5$. Due to lack of the amount of industrial exhaust emission data, here GM $(1,1)$ algorithm is utilized to pretreat corresponding data in 2006 and 2011. Then, the amount of industrial exhaust emission data in 2006 and 2011 are respectively 42535 and 69335 . The original data is standardized by formula (1), and the grey correlation degree computed by the grey correlation method are listed in Table.2.

Table 2. The grey correlation degree.

\begin{tabular}{ccccc}
\hline PM10 & $\mathrm{SO}_{2}$ & $\mathrm{NO}_{2}$ & OvallIEI & TotalIEE \\
\hline Ods 0.8569 & 0.4674 & 0.4831 & 0.6841 & 0.4440 \\
\hline
\end{tabular}

*Ods:The original data sequence; ;

OvallIEI :The overall investment for environment improvement (millions);

* TotalIEE : Total of industrial exhaust emission (billion cubic meters) .

According to the data in Table.2, we get the main factors affecting the air quality of Shijiazhuang city in Hebei is $P M 10$ and the overall investment for environment improvement, followed by $\mathrm{SO}_{2}$, $\mathrm{NO}_{2}$ and the total of industrial exhaust emission. 


\section{THE SIMULATION AND PREDICTION OF THE AIR QUALITY BASED ON $G M(1,1)$}

\section{The $G M(1,1)$ model}

The gray system theory $G M(1,1)$ is introduced by the following four procedures ${ }^{[1]}$.

I)1---AGO (Accumulated Generating Operator)

Constructing the original data sequence

$x^{(0)}=\left(x^{(0)}(1), x^{(0)}(2), \ldots, x^{(0)}(n)\right)$

and accumulating the original data sequence

$x^{(1)}=\left(x^{(1)}(1), x^{(1)}(2), \ldots, x^{(1)}(n)\right)$;

where $x^{(1)}(k)=\sum_{i=1}^{k} x^{(0)}(i) \quad(k=1,2,3, \mathrm{~L}, n)$.

$x^{(1)}(k)=x^{(1)}(k-1)+x^{(0)}(k), \quad k=1,2,3, \ldots, n$.

The original data sequence can satisfy

$\frac{x^{(0)}(k)}{x^{(1)}(k-1)}<\varepsilon_{i} \quad, k=1,2,3, \ldots, n$.

$\left\{\varepsilon_{i}\right\}$ is a decreasing sequence

II)Constructing a differential equation $(\operatorname{GM}(1,1))$ with one variable. Assuming

$B=\left(\begin{array}{cc}-\frac{1}{2}\left(x^{(1)}(1)+x^{(1)}(2)\right) & 1 \\ -\frac{1}{2}\left(x^{(1)}(2)+x^{(1)}(3)\right) & 1 \\ -\frac{1}{2}\left(x^{(1)}(3)+x^{(1)}(4)\right) & 1 \\ M & M \\ -\frac{1}{2}\left(x^{(1)}(n-1)+x^{(1)}(n)\right) & 1\end{array}\right) \quad Y_{N}=\left(x^{(0)}(2), x^{(0)}(3), x^{(0)}(4), \ldots, x^{(0)}(n)\right)^{T}$.

$x^{(1)}(k)$ satisfies the differential equation, and sequentially the $G M(1,1)$ model is

$\frac{d x^{(1)}}{d t}+a x^{(1)}=b$,

By using the least square method, the estimated value of $a$ and $b$ in Eq.(2) are $\hat{a}=\left(B^{T} B\right)^{-1} \cdot B^{T} \cdot Y_{N}=(a, b)^{T}$.

By putting $\hat{a}$ into Eq.(2), the solution of the equation is

$\hat{x}^{(1)}(k+1)=\left(x^{(0)}(1)-\frac{b}{a}\right) \cdot e^{-a k}+\frac{b}{a}, \quad(k=1,2,3, \ldots, n)$.

and namely

$\hat{x}^{(0)}(k)=\hat{x}^{(1)}(k+1)-\hat{x}^{(1)}(k)=\left(1-e^{a}\right)\left[x^{(0)}(1)-\frac{b}{a}\right] \cdot e^{-a k}$.

III)Finding $\hat{x}^{(0)}(k+1)$

$\hat{x}^{(0)}{ }_{(k+1)}=\hat{x}^{(1)}(k+1)-\hat{x}^{(1)}(k),(k=1,2,3, \ldots, n)$.

Because standardized data sequence is achieved by Eq.(1), and the fitting data should be transformed into the original form.

$\hat{Y}(k)=(M-m) \hat{x}^{(0)}(k)+m, \quad(k=1,2,3, \ldots, n)$. 


\section{The prediction using the $G M(1,1)$ model}

First, the original standardized data, or the date number of air quality below the second level in Shijiazhuang city from 2006 to 2011, are processed by Eq.(1) and the standardized sequence is

$X^{(0)}=[1.0000,0.9394,0.6061,0.0606,0.0303,0.0000]$;

$\frac{x^{(0)}(k)}{x^{(1)}(k-1)}=[0.0000,1.9394,1.3125,1.0238,1.0116,1.0000]$

When $k>3, \frac{x^{(0)}(k)}{x^{(1)}(k-1)}<\varepsilon_{i} \quad$ can be satisfy.

By formulating $\operatorname{GM}(1,1)$ model to predict the data in the next two years, we arrive that: $a=0.8158, b=2.2111$.

Thus, $x^{(0)}(k)=2.7103-1.7103 \cdot e^{-0.8158(k-1)}$,

$k=1,2,3, \ldots, n$.

By solving the equation, the fitting results are

$$
x^{(0)}=[1.0,0.954,0.422,0.187,0.083,0.037,0.016,0.007] .
$$

Because standardized data sequence is achieved by (1), and the fitting data should be transformed into the original form.

$\hat{Y}=[78.0,76.5,58.9,51.25,47.7,46.2,45.5,45.2]$.

The day number of air quality below the second level in Shijiazhuang city of Hebei in 2012 and 2013 are 45.5, and 45.2. Because one day represents 24 hours, 0.5 day is 12 hours and 0.2 day is 4.8 hours. The comparison between the origin data sequence and the predicted data sequence are shown in Table.3.

From the perspective of the average relative error, the precision of the $G M(1,1)$ model is not high and the average relative error is equal to 0.05 . However, this result satisfies the precision standard of the second model, therefore it can be made relatively longer time prediction.

By using the $G M(1,1)$ model to predict the day number of air quality below the second level of Shijiazhuang city in Hebei, the day numbers are 45. The simulation for day number of air quality below the second level in Shijiazhuang city of Hebei from 2007 to 2011 and origin data are shown in Table.3.

Table 3. Simulation and prediction results using $G M(1,1)$ model.

\begin{tabular}{llcrl}
\hline Time & Ods & Sis & Residual & Relative er \\
\hline 2007 & 76 & 76.4773 & -0.4773 & 0.0063 \\
2008 & 65 & 58.9219 & 6.0781 & 0.0935 \\
2009 & 47 & 51.1575 & -4.1575 & 0.0885 \\
2010 & 46 & 47.7233 & -1.7233 & 0.0375 \\
2011 & 45 & 46.2045 & -1.2045 & 0.0268 \\
& & & & \\
\hline 2012 & --- & 45.5327 & ---- & \\
2013 & --- & 45.2356 & ---- & \\
& & & & 0.0505 \\
\hline Average relative error & & \\
\hline
\end{tabular}

*Ods:The original data sequence;

*Sis:Simulated sequence; 


\section{THE DISCUSSION AND CONCLUSIONS}

By modeling atmosphere pollution in Hebei and employing the grey theory, we get the main factors affecting the air quality of Shijiazhuang city in Hebei is $P M 10$ and the overall investment for environment improvement, followed by $\mathrm{SO}_{2}, \mathrm{NO}_{2}$ and the total of industrial exhaust emission.

In order to achieve further accomplishment, we arrive the next three advises by the model discussed above.

\section{To lower the amount of inhalable particle in the air}

The concentration of inhalable particles determines the atmospheric quality by the mathematical results of the model. In order to alleviate the atmospheric pollution, reducing the inhalable particles is the top primary.

a). To Restrict Industrial exhaust emission

Industrial exhaust should be well treated. Dust exhaust must be processed and then carefully released in the atmosphere. Automobile exhaust, the installment of processing device of automobile is strongly advised The relevant law and regulation must be fully fulfilled. Using asphalt road can decrease the dust raised by pack way.

b). To decrease the concentration of the inhalable particle

Regular road clearing is essential. Planting shall also receive attention. the development of alternative energy should paced up.

\section{To efficiently control and manage the gas emission}

The principal pollutants consist of sulfur dioxide and compounds of nitrogen and oxygen. Additionally, the reduced issue of industrial exhaust leads to the decreased concentration of each ingredient in industrial exhaust, thereby reducing air pollution levels. In order to moderate the gas emission, the gas should be well treated before being released. Moreover, searching alternatives for traditional energy should been taken into consideration and simultaneously minimizing using fossil energy.

\section{To significantly enhance control efforts and cooperation among the regions}

The region cooperation among Beijing, Tianjing and Hebei is the foundation for unified control, surveillance and planning to improve the air quality and to better ecological atmosphere.

\section{ACKNOWLEDGEMENT}

This research work was supported by the foundation for the key project of science and technology of Agricultural University of Hebei (NO.ZD201508).

\section{REFERENCES}

[1] Dang Yao-guo, Liu Si-feng, Wang Zheng-xin, et al. Grey forecasting and decision making model research. Beijing: Science Press, 2009.

[2] Dai Wei, Qu Dong. Prediction of Air Pollutants SO2 in Qinhuangdao based on the GM(1,1)Model. Journal of EMCC [J]. 2011,21(1):55-57.

[3] XU Song, CHENG Tong-qing. Application Grey System Theory to Forecasting and Analysis on Affectec Factors of Atmospheric NO2 Concentration. Journal of Nanchang University[J].

2006,30(1):99-102.

[4] Feng Jia-wu,Peng Xiao-wu,Xu Zhen-cheng,et al. Analysis of haze pollution level over pearl river delta during 2005-2009 [J]. Environmental Science \& Technology,2012,35(11):75-78.

[5] Yu X. G.,Wu J. et al. Study progress on the correlation of atmospheric haze and particulates [J]. Environmental Pollution \& Control, 2010,32(2):86-90.

[6] Wu D,Deng X. J. et al. Study on the visibility reduction caused by atmospheric haze in Guangzhou area [J]. Journal of Tropical Meteorology, 2007,23(1):1-6.

[7] Wu Dui. Formation and evolution of haze weather [J]. Environmental Science \& Technology, 2011, 34(3):157-162. 
International Forum on Energy, Environment Science and Materials (IFEESM 2015)

[8] $\mathrm{Wu} \mathrm{D}, \mathrm{Wu} \mathrm{C}$,et al. Air pollution episode in southern China due to the long range transport of coarse particle aerosol [J]. China Environmental Science, 2011,31(4):540-545. 